\title{
IBM ILOG Graph Layout for Eclipse
}

\author{
Jerome Joubert, Stephane Lizeray, Romain Raugi, and Georg Sander \\ IBM ILOG Visualization Group \\ $\{j \cdot$ joubert, lizeray, romain.raugi\}@fr.ibm.com, georg.sander@de.ibm.com
}

\section{Introduction}

Eclipse is becoming increasingly popular within the Java developers' community, and with the availability of RCP (Rich Client Platform), Eclipse is also seen as a very attractive framework for building professional stand-alone applications. For creating visual interfaces and diagram displays, Eclipse provides the Graphical Modeling Framework (GMF) and the Graphical Editing Framework (GEF). Both frameworks provide only very simple support for automatic graph layout natively but lack professional quality layout capabilities. IBM ILOG JViews Graph Layout for Eclipse fills this gap. It brings a concrete solution to developers who need to produce professional diagram visualization and modeling on Eclipse. It contains a wide collection of layout algorithms and configuration services, but also interacts well with GEF and GMF. The loose coupling of the application architecture and the graph layout provides last-minute integration capabilities giving a chance to incorporate layout services very late in the development process.

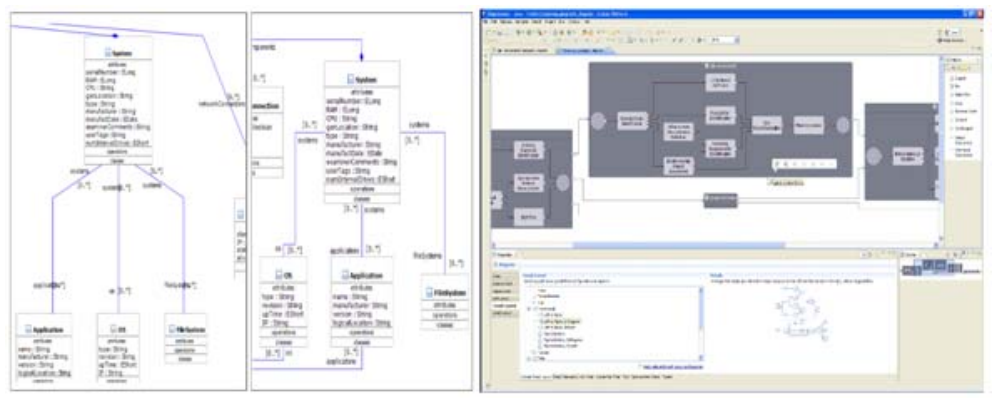

Fig. 1. Left: UML Diagram, right: Eclipse Application made with IBM ILOG Graph Layout for Eclipse

\section{Highlights}

IBM ILOG JViews Graph Layout for Eclipse provides advanced algorithms to automatically arrange diagrams so that they are readable by a human being. There are different types of algorithm. Some optimize node and link placement, some route links in order to minimize crossings, some compute text label placement with minimal overlap. The following families of algorithms are available: 
- Normal tree layouts, Org chart layouts, Radial tree layouts

- Hierarchical layouts

- Spring Embedder

- Bus layout

- Topological mesh layout

- Circular layout

- Recursive layout for nested graphs

- Label layout, annotation layout

- Link routing (several algorithms and variants)

- Improved display of link crossings

The layout services are available as different Eclipse plug-ins. The plug-in based on GEF delivers graph layout core capabilities via an adapted graph model that issues GEF requests and triggers GEF commands to position nodes and links during layout. This is the natural, nondestructive approach for GEF. This architecture ensures that layout is undoable and persistent. The ILOG JViews Graph Model for Eclipse hides the complexity and can easily be attached to any GEF Graph Editing Part. Similarly, the plug-in based on GMF utilizes all GEF services and additionally provides support for the modelling capabilities of EMF. An animation framework and various predefined graphic shapes (subgraph shapes, links with crossing jogs) complete the services offered by IBM ILOG Graph Layout for Eclipse.
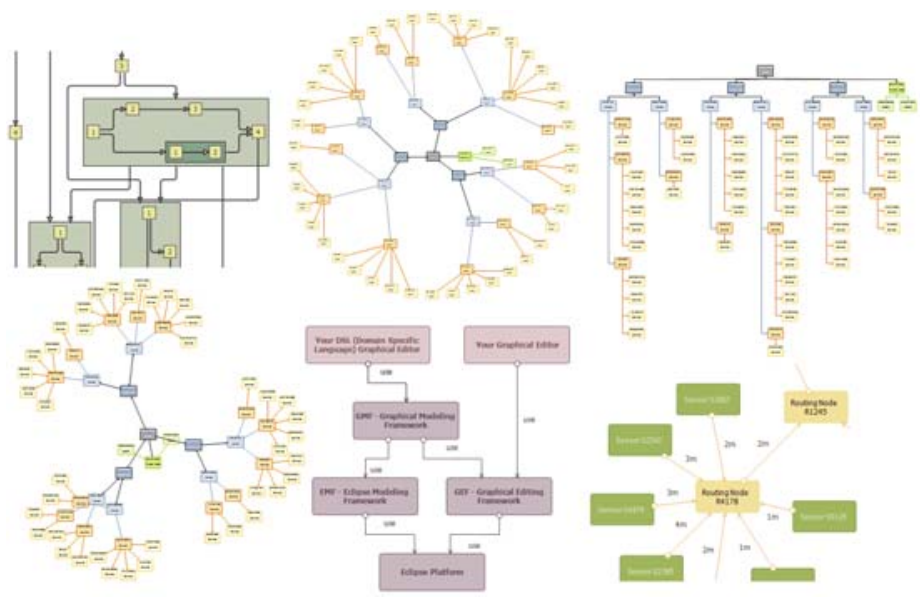

Fig. 2. Sample Layouts of IBM ILOG Graph Layout for Eclipse 